\title{
Thermal battery pole piece uniform density testing research
}

\author{
Wenshi Ren, Tianshu Zhang,Zhenghua Feng \\ School of China Three Gorges University, Yichang 443000, China; \\ School of China Three Gorges University, Yichang 443000, China; \\ School of China Three Gorges University, Yichang 443000, China; \\ 936421018@qq.com,892585112@126.com,65085328@qq.com
}

Keywords: Thermal battery,Density monitoring.

\begin{abstract}
The most prominent feature of the thermal battery is the high discharge current density,especially pulse discharge,which has high specific energy and power; followed by a short activation time, very flexible to use, this is unmatched by other battery; followed by the ability harsh environmental conditions, particularly the use of a wide temperature range.Therefore, thermal batteries appeared, favored by the military,first, put it to use in weapons. Thermal battery pole piece during the production process of the pole pieces, uneven density defects seriously affect the thermal and electrical properties of its runtime.In this paper, through the thermal battery pole piece uniformity of eddy current test in order to achieve thermal battery pole piece density of conformity assessment, which can ensure the product quality of the thermal battery.
\end{abstract}

\section{Introduction}

At present, many advanced high-tech weapons in the world put thermal batteries as their preferred source of power,involved in the variety of weapons, including a variety of tactical missiles, strategic missiles, cruise missiles, guided missiles, nuclear weapons, all kinds of advanced bombs, artillery, aircraft, space vehicles and underwater weapons, etc.Thermal batteries in these types of weapons, some used as fuses and detonator,such as proximity bomb fuse, electric squib detonators detonated;some for ignition, such as missile launch, the igniter for the propellant;some used as the control system and telemetry system power;ProBook 4415s HP batteries are used directly as the propulsion power of weapons, such as underwater torpedo propulsion power supply;some used as life-saving power supply, such as aircraft ejection seat power supply and so on.In short, the development of modern sophisticated weapons, is inseparable from the development of thermal batteries. In addition to thermal batteries in the military field has its special uses, in civilian areas have also been emerging trends,although this area has not been done in-depth research to develop a detailed application, such as fire power, high temperature prospecting underground power supply should have its foothold.RT(RT: Radiographic Testing)、UT(UT: Ultrasonic Testing) 、 MT(MT:Magnetic Particle Testing)、PT(PT:Penetrant Testing)and called the five NDT(NDT: Non-Destructive Technology).Eddy current testing compared with ray detector, the human body without radiation hazards, and can work in extremely harsh environments;compared with ultrasonic testing, no coupling agent;compared with magnetic particle testing, it can be used for any conductive medium (ferromagnetic, diamagnetic substance, paramagnetic);compared with the penetration testing, it can not only detect the surface openings of the components, but also can detect the internal defects.For defects occurring in the multilayer structure, eddy current testing has unique advantages.Therefore, eddy current testing of aerospace, petrochemical, transportation, energy, power plant construction and ship construction and other departments widely used. 


\section{Basic theory of eddy current testing}

\subsection{Eddy Current Testing Simplified Model}

Eddy current testing model has many problems, the design concept is only related to calculation of the coil impedance of the top two conductive structure,as shown in figure 2.4:

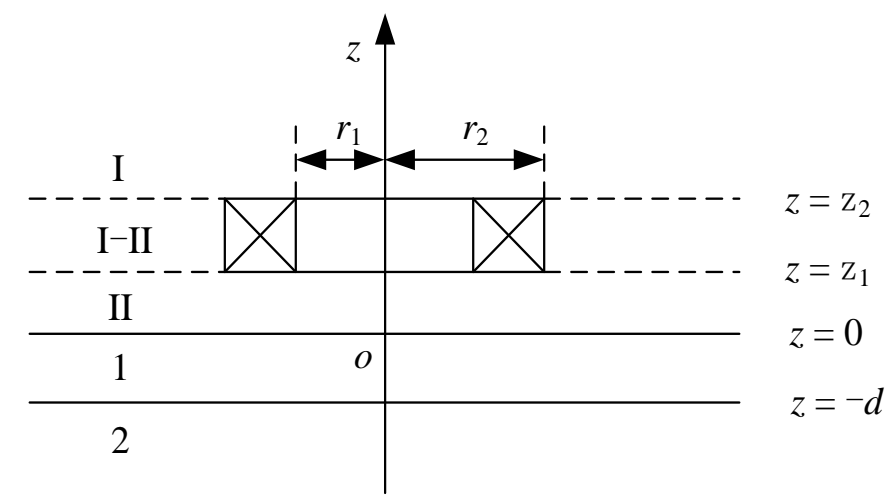

Fig. 2.4 two conductive structure located above the coil

When in the 1960s, Dodd and others in the probe is positioned above the conductive material probe impedance simulation to study the introduction of the magnetic vector potential.They derive a mathematical equation for the probe impedance of the probe located at the top of the double layer conductive material.In order to make the relationship between the impedance and phase research more convenient, the equation will make the following changes:

$Z=K \int_{0}^{\infty} \frac{1}{\lambda_{0}^{5}} I^{2}\left(r_{2}, r_{1}\right)\left\{2\left(z_{2}-z_{1}\right)+\lambda_{0}^{-1}\left[2 e^{-\lambda_{0}\left(z_{2}-z_{1}\right)}-2+A\left(\lambda_{0}\right) R\left(\lambda_{0}\right)\right]\right\} d \lambda_{0}$

Form in: $K=\frac{j \omega \pi \mu_{0} N^{2}}{\left(z_{2}-z_{1}\right)^{2}\left(r_{2}-r_{1}\right)^{2}}, \quad P\left(\lambda_{0}\right)=\left(e^{-\lambda_{0} z_{1}}-e^{-\lambda_{0} z_{2}}\right)^{2}$,

$R\left(\lambda_{0}\right)=\frac{\left(\lambda_{1}+\lambda_{0}\right)\left(\lambda_{1}-\lambda_{0}\right)-\left(\lambda_{1}+\lambda_{0}\right)\left(\lambda_{1}-\lambda_{0}\right) e^{2 \lambda_{1} d}}{\left(\lambda_{0}-\lambda_{1}\right)\left(\lambda_{1}-\lambda_{0}\right)+\left(\lambda_{1}+\lambda_{0}\right)\left(\lambda_{1}+\lambda_{0}\right) e^{2 \lambda_{1} d}}, \quad I\left(\lambda_{0}\right)=\int_{\lambda_{0} r_{1}}^{\lambda_{0} r_{2}} x J_{1}(x) d x$,

$\lambda_{1}=\sqrt{\lambda_{0}^{2}+j \omega \sigma \mu}$,

Wherein, $\mathrm{Z}$ is the total impedance value of the probe; $\mathrm{J}$ is the imaginary unit; $\omega$ is the angular frequency; $n$ is the density of turns of the coil; $r_{1}$ is the inner diameter of the coil; $r_{2}$ is the outer diameter of the coil; $z_{1}$ is the probe lift off distance; $z_{2}$ is on the upper part of the coil to the metal plate surface distance; $\lambda_{0}$ is the integral variable; $J_{1}(x)$ is the first order Bessel function of the first kind, $\mu$ is the magnetic permeability of the material, $\sigma$ for the environment of the material conductivity, $\mathrm{d}$ is the thickness of metal materials.

The expression (2.8) can be analyzed with the change of eddy current parameters, and the experimental results can be achieved.For example, the electrical conductivity, thickness, permeability, and the total impedance of the coil between the contact.By using this model, we can also analyze the interference caused by the factors such as the weakening of the edge and the lift off effect.

\subsection{Eddy current skin effect}

Based on the principle of plane electromagnetic wave, can draw the electric eddy current detection in depth of skin: $\delta=\frac{1}{\sqrt{\pi f \mu \sigma}}$ 
Where $\delta$ is the depth of ingratiate oneself, defined as the magnitude of the magnetic field intensity and the current density decreased to $36.7 \%$ on the surface corresponding to the $f$ is the excitation frequency, ${ }^{\mu}$ for conductive material permeability, $\sigma$ is the conductivity of conductive material.

By the above formula, it is easy to see, the excitation frequency, magnetic permeability, conductivity determines the eddy current detection ingratiate oneself depth.In a typical application, the material to be detected magnetic and electrical conductivity are usually not change, then you can not consider these data, only the excitation frequency will affect the depth ingratiate oneself.It can be obviously seen, excitation frequency and ingratiate oneself depth showed a negative correlation, that is the incentive, the lower the frequency, ingratiate oneself depth is deeper.In order to study the defects of the deeper, only choose to reach the excitation frequency depth to ingratiate oneself.

According to Faraday's law of electromagnetic induction, when the excitation voltage is sinusoidal AC signal, the inductive voltage of the coil eddy current detection probe can be expressed as follows: $V=-j \omega N \pi r^{2} B$

In the formula, $V$ is the induction voltage of the coil, the $\omega$ is the angular frequency, the $N$ is the number of turns of the coil, the $r$ is the radius of the coil, and $B$ indicates the magnitude of the magnetic induction intensity of the coil.Among them, $\omega=2 \pi f$. Can clearly be seen in (2.10), the amplitude of the voltage induced excitation frequency was positively correlated, that is, the sensitivity of the probe with the excitation frequency coil is also a positive correlation.

Therefore, when the defect is detected multilayer conductive structures, always reduce the excitation frequency. The problem is that this process, when using a coil probe detection sensitivity of the sensor will decrease.To solve this problem, you can use the following methods:

1)increase the radius of the inner and outer coils;

2) Increase the number of turns;

3) The change of magnetic induction intensity is detected by using the sensor sensitivity is not affected by the excitation frequency;

4) By the addition of high-power excitation coil, improving the magnetic flux density $B$, to compensate for reduced excitation voltage amplitude.

\section{Eddy Current simulation program}

\subsection{Cylindrical hollow coil impedance model}

Section 2.2 gives the analytical expression of the impedance conductive coil located above the two layers of flat Dodd established.Generally, the expression can be divided into two parts:Coil impedance variation $\Delta Z$ and the impedance of the coil in the air $Z_{\text {air }}$.Therefore, the total impedance expression of the coil can be re expressed as follows: $Z=Z_{\text {air }}+\Delta Z$

Among them,

$$
\begin{aligned}
Z_{\text {air }} & =\frac{j 2 \pi \omega \mu_{0} N_{c}^{2}}{\left(r_{2}-r_{1}\right)^{2}\left(z_{2}-z_{1}\right)^{2}} \int_{0}^{\infty} \frac{\chi^{2}\left(\lambda_{0} r_{1}, \lambda_{0} r_{2}\right)}{\lambda_{0}^{6}}\left[\lambda_{0}\left(z_{2}-z_{1}\right)+e^{-\lambda_{0}\left(z_{2}-z_{1}\right)}-1\right] d \lambda_{0} \\
& =2 K \int_{0}^{\infty} \frac{I^{2}}{\lambda_{0}^{6}}\left[\lambda_{0}\left(z_{2}-z_{1}\right)+e^{-\lambda_{0}\left(z_{2}-z_{1}\right)}-1\right] d \lambda_{0} \\
\Delta Z & =\frac{j \pi \omega \mu_{0} N_{c}^{2}}{\left(r_{2}-r_{1}\right)^{2}\left(z_{2}-z_{1}\right)^{2}} \int_{0}^{\infty} \frac{\chi^{2}\left(\lambda_{0} r_{1}, \lambda_{0} r_{2}\right)}{\lambda_{0}^{6}}\left(e^{-\lambda_{0} z_{1}}-e^{-\lambda_{0} z_{2}}\right)^{2} R\left(\lambda_{0}\right) d \lambda_{0} \\
& =K \int_{0}^{\infty} \frac{I^{2}}{\lambda_{0}^{6}} P^{2} R\left(\lambda_{0}\right) d \lambda_{0}
\end{aligned}
$$


From formula (3.2) and (3.3) it can be known that although the product function in the formula is complex, it can be divided into several parts.Among the parameters K, I, P, R is the key.

\subsection{Trapezoidal integration algorithm}

Calculation of integral: $I(f)=\int_{a}^{\mathrm{b}} f(x) d x$

The core idea of the approximate formula of it is to replace the $\mathrm{f}(\mathrm{x})$ with an approximate function of the integral approximation.

$\mathrm{F}(\mathrm{x})$ linear interpolation polynomial is: $P_{1}(x)=\frac{(b-x) f(a)+(x-a) f(b)}{b-a}$

Among them, the interpolation nodes are a and b. $P_{1}(x)$ in the interval [a, b] on the integral value is equal to the shadow part of the shadow of the area, its value is

$$
T_{1}(f)=(b-a)\left[\frac{f(a)+f(b)}{2}\right]
$$

When $\mathrm{f}(\mathrm{x})$ is in the interval $[\mathrm{a}, \mathrm{b}]$ is similar to the linear function, the value of the integral $I(f)$ can be estimated by this method.

When using multiple length of the sub range, we can use as a general formula to simplify the calculation, set $\mathrm{n}$ to represent the number of sub intervals, so that the length of each sub range $\boldsymbol{h}=\frac{\boldsymbol{b}-\boldsymbol{a}}{\boldsymbol{n}}$, sub range of the endpoint is $x_{j}=a+j h, j=0,1, \cdots, n$

Split the original score into $n$ integral.

$$
I(f)=\int_{\mathrm{a}}^{\mathrm{b}} f(x) d x=\int_{x_{0}}^{x_{n}} d x=\int_{x_{0}}^{x_{1}} f(x) d x+\int_{x_{1}}^{x_{2}} f(x) d x+?+\int_{x_{n-1}}^{x_{n}} f(x) d x
$$

Use (4.4) the calculation of each integral,And pay attention to each sub-interval [a, b] of length h,there

$$
\begin{aligned}
& I(f) \approx h\left[\frac{f(0)+f(1)}{2}\right]+h\left[\frac{f(1)+f(2)}{2}\right]+h\left[\frac{f(2)+f(3)}{2}\right]+ \\
& \cdots+h\left[\frac{f\left(x_{n-1}\right)+f\left(x_{n}\right)}{2}\right]
\end{aligned}
$$

Can get more concise form: $\quad T_{n}(f)=h\left[\frac{1}{2} f\left(x_{0}\right)+f\left(x_{1}\right)+f\left(x_{2}\right)+\cdots+f\left(x_{\mathrm{n}-1}\right)+\frac{1}{2} f\left(x_{n}\right)\right]$

This method is called trapezoidal numerical integration method, and the subscript $\mathrm{n}$ indicates the number of sub ranges, $x_{0}, x_{1}, \cdots, x_{n}$ is called the node of numerical integration.

\subsection{Flow chart of coil impedance calculation}

Air coil impedance calculation program flow shown in Figure 3.1. 


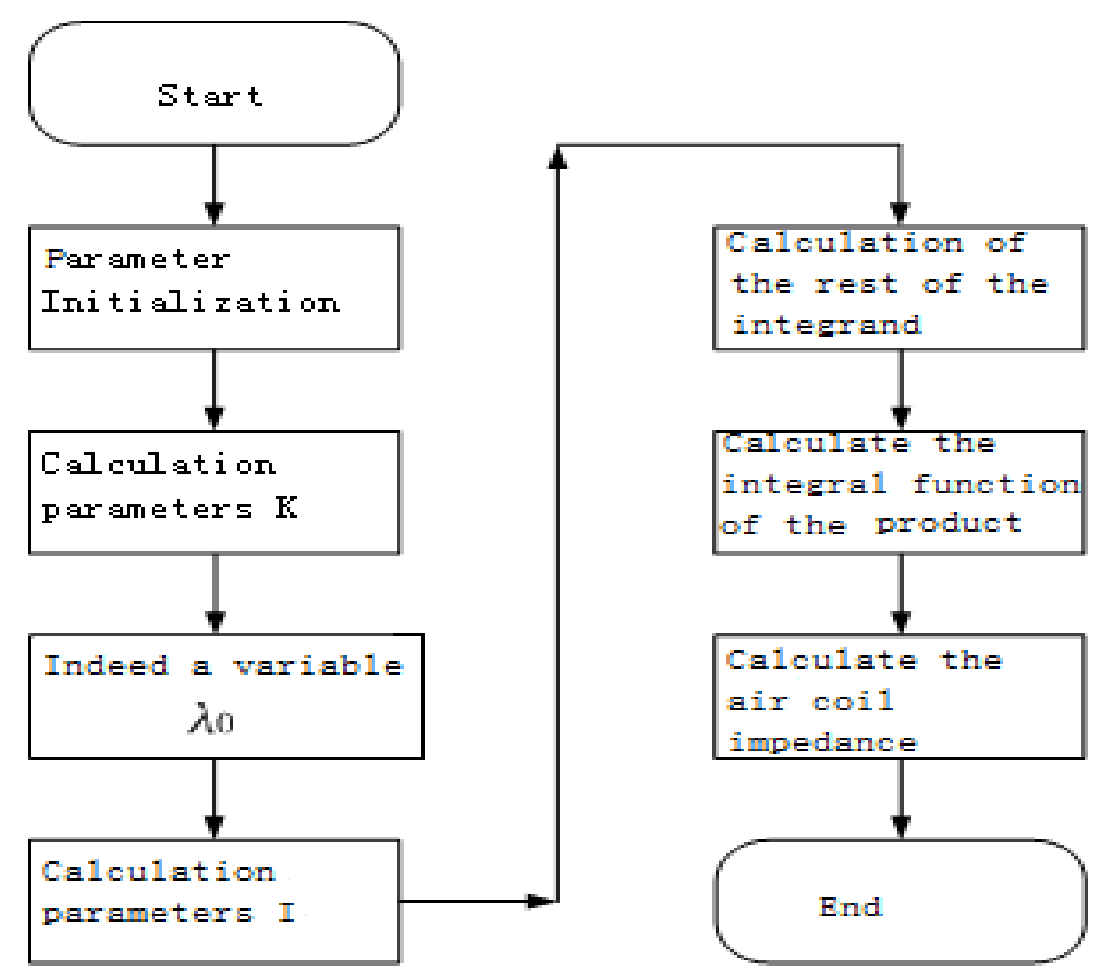

Fig. 3.1 air coil impedance calculation program flow chart

Coil impedance variation calculation program flow shown in Figure 3.2.

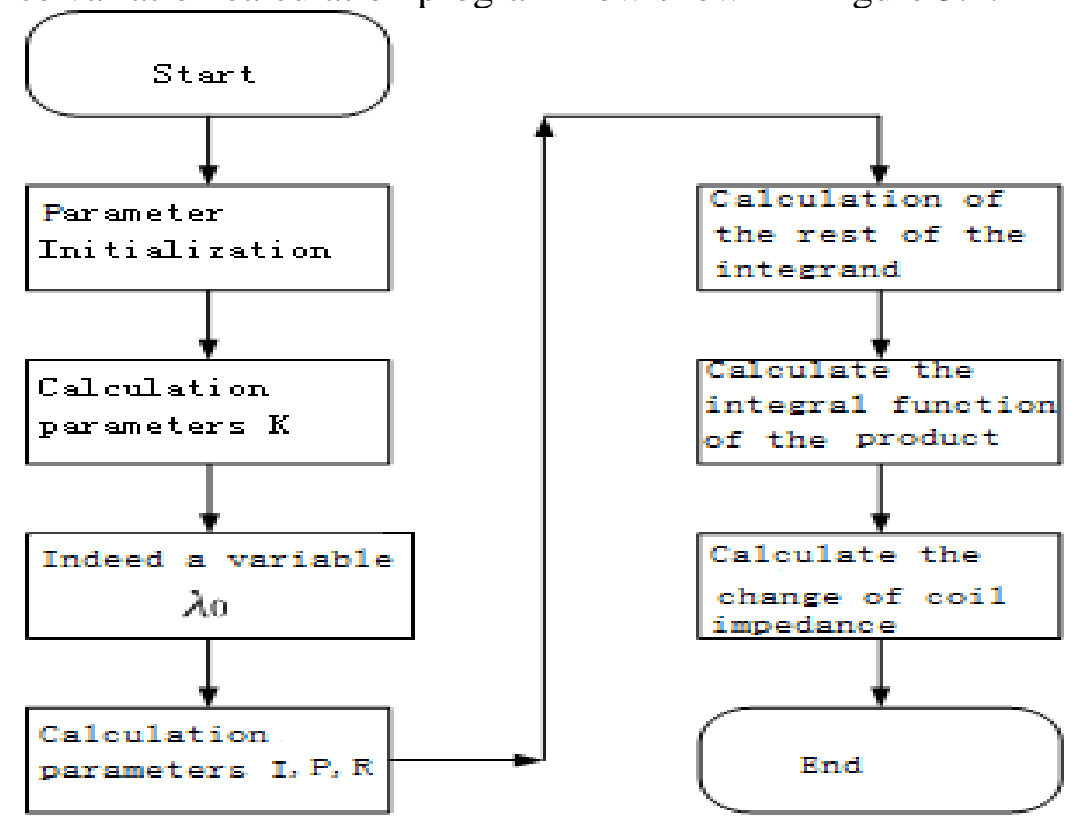

Fig. 3.2 coil impedance change amount calculation program flow chart

\subsection{Specific modules impedance calculation program implementation}

Parameter initialization is to give the initial value of the variables in the program.The specific program initialization parameters are as follows:

1. The initialization parameters of the program of the coil impedance in the air are as follows: The coil diameter of $r_{1}$ is $4.0 \mathrm{~mm}$, the diameter of the coil $r_{2}$ is $8.0 \mathrm{~mm}$, and the number of turns of the coil $N$ is 900,lift off height $z_{1}=0.5 \mathrm{~mm}, z_{2}=2.5 \mathrm{~mm}$, vacuum permeability $\mu_{0}=4 \pi * 10^{-7} \mathrm{H} / \mathrm{m}$,material selection of a certain type of thermal battery cathode plate, $\sigma=3.54 * 10^{7} \mathrm{~S} / \mathrm{m}$, excitation frequency $f=1000 \mathrm{HZ}$.In the program, PI said $\pi$, mu0 expressed $\mu_{0}$, omig said $\omega$, Alfa said $\lambda_{0}$, sigma said $\sigma$ (conductivity).By using the formula of the 
second chapter and the program design of the third chapter, we can get the following kinds of condition to detect the value of the coil impedance.The influence of the thickness of the battery pole piece on the coil under different lift off height is obtained.

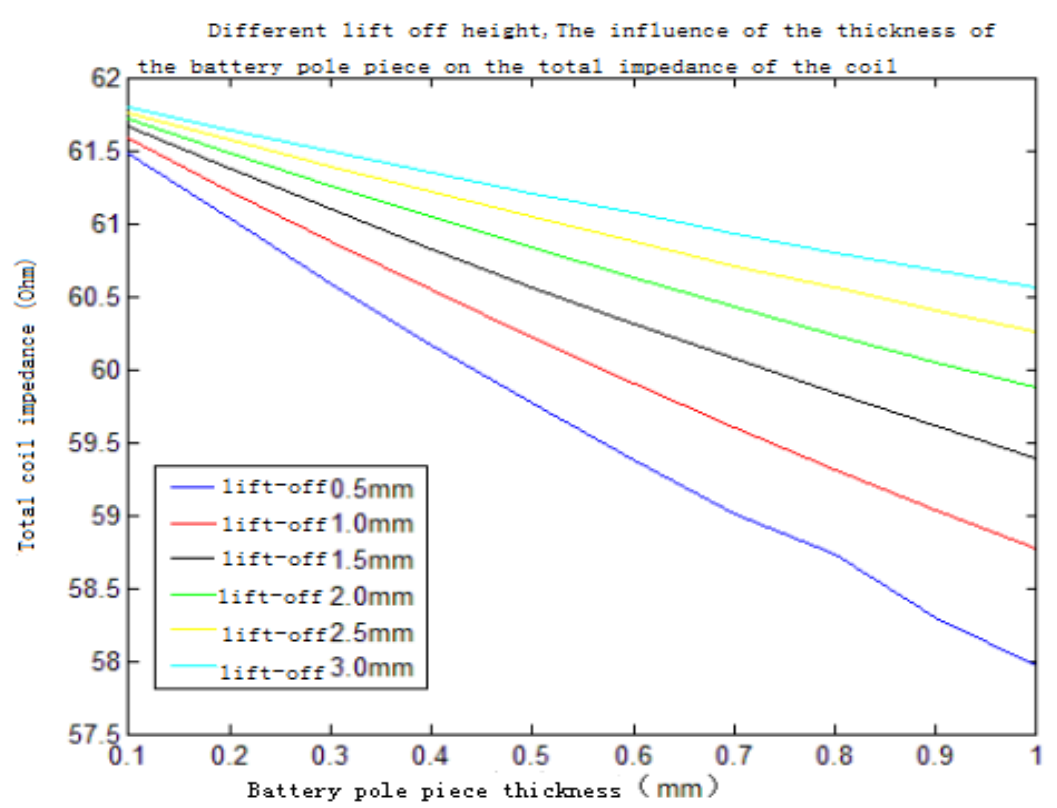

Fig. 3.3

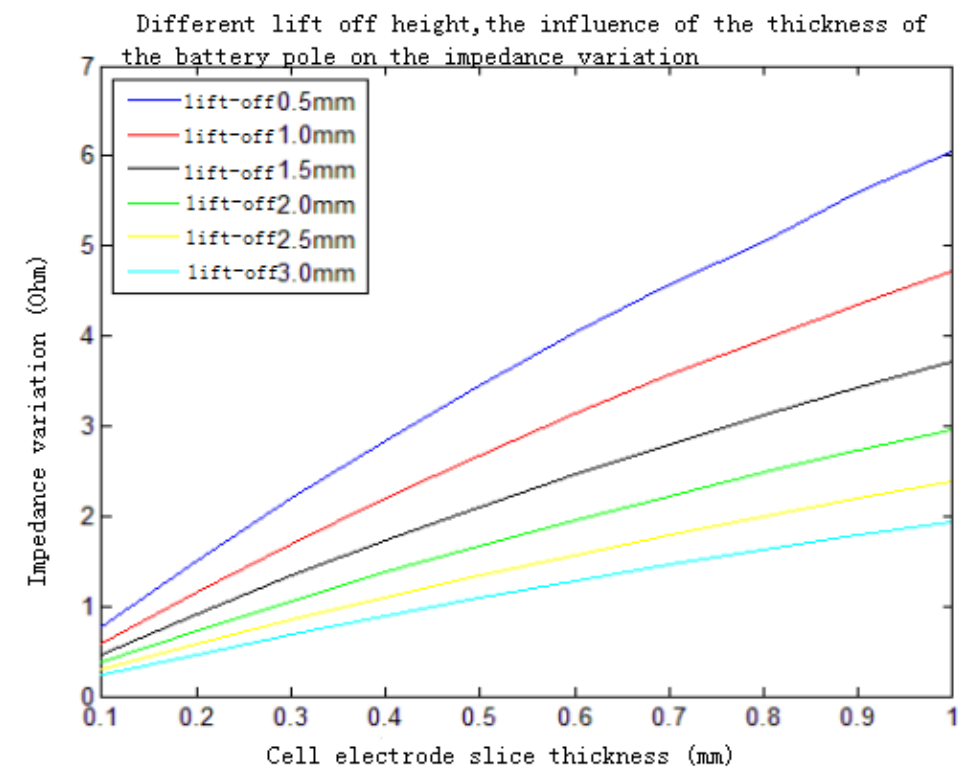

Fig. 3.4

Can be seen from figure 3.3 and figure 3.4,the total impedance of the coil decreases with the increase of the thickness of the battery pole piece,and the change of the coil impedance increases with the increase of the thickness of the battery pole piece.But in different lift height, the change of the coil impedance is different. When the lift off height is high, the variation of the coil impedance increases with the increase of the thickness of the metal sheet,the degree of change is small, which is good for the measurement of data; when the lift off height is low, the variation of the coil impedance increases with the increase of the thickness of the metal sheet,change degree is bigger, the deviation of data measurement is bigger.So in the experiment, the data can be improved by increasing the height of lifting, which makes the measurement record of data more accurate. 


\section{The influence of the thickness of the battery pole piece at different frequencies on the coil}

(2.1) Set the input signal frequency is $1000 \mathrm{~Hz}$, the metal thickness is $\mathrm{d}=0.1 \mathrm{~mm}-1.0 \mathrm{~mm}$, lift off height is $1 \mathrm{~mm}$, the upper limit of the integral is 3000 .

(2.2) Set the input signal frequency is $1500 \mathrm{hz}$, the metal thickness is $\mathrm{d}=0.1 \mathrm{~mm}-1.0 \mathrm{~mm}$, lift off height is $1 \mathrm{~mm}$, the upper limit of the integral is 3000 .

(2.3) Set the input signal frequency is $2000 \mathrm{~Hz}$, the metal thickness is $\mathrm{d}=0.1 \mathrm{~mm}-1.0 \mathrm{~mm}$, lift off height is $1 \mathrm{~mm}$, the upper limit of the integral is 3000 .

(2.4) Set the input signal frequency is $2500 \mathrm{~Hz}$, the metal thickness is $\mathrm{d}=0.1 \mathrm{~mm}-1.0 \mathrm{~mm}$, lift off height is $1 \mathrm{~mm}$, the upper limit of the integral is 3000 .

(2.5) Set the input signal frequency is $3000 \mathrm{hz}$, the metal thickness is $\mathrm{d}=0.1 \mathrm{~mm}-1.0 \mathrm{~mm}$, lift off height is $1 \mathrm{~mm}$, the upper limit of the integral is 3000 .

(2.6) Set the input signal frequency is $3500 \mathrm{hz}$, the metal thickness is $\mathrm{d}=0.1 \mathrm{~mm}-1.0 \mathrm{~mm}$, lift off height is $1 \mathrm{~mm}$, the upper limit of the integral is 3000 .

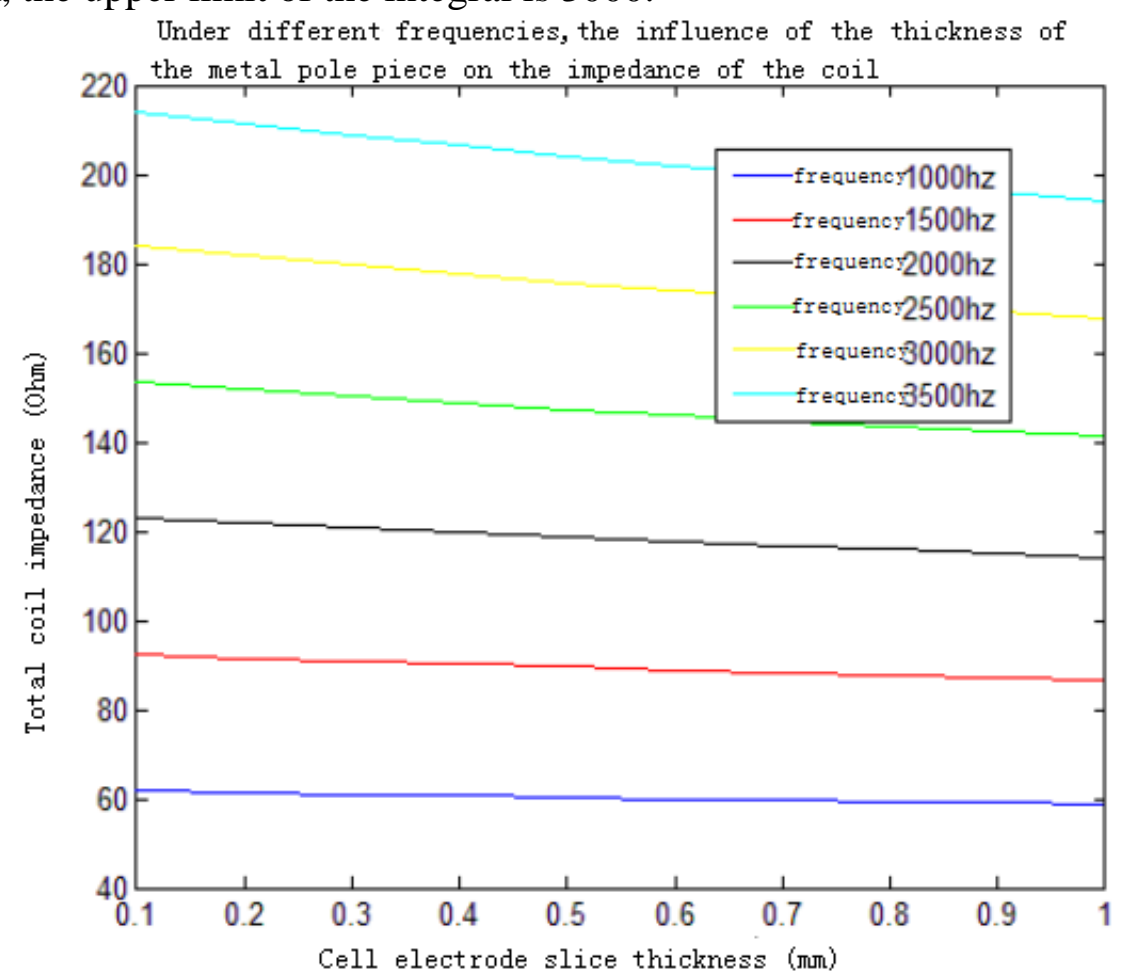

Fig. 3.5

Can be seen from figure 3.5,the total impedance of the coil decreases with the increase of the thickness of the battery pole piece. When the excitation frequency is high, the total impedance of the coil is larger, and the record is relatively easy to be measured;when the excitation frequency is low, the total impedance of the coil is small, which is not conducive to the recording of the data.So in the experiment, we can improve the convenience of data measurement by appropriately increasing the excitation frequency. 


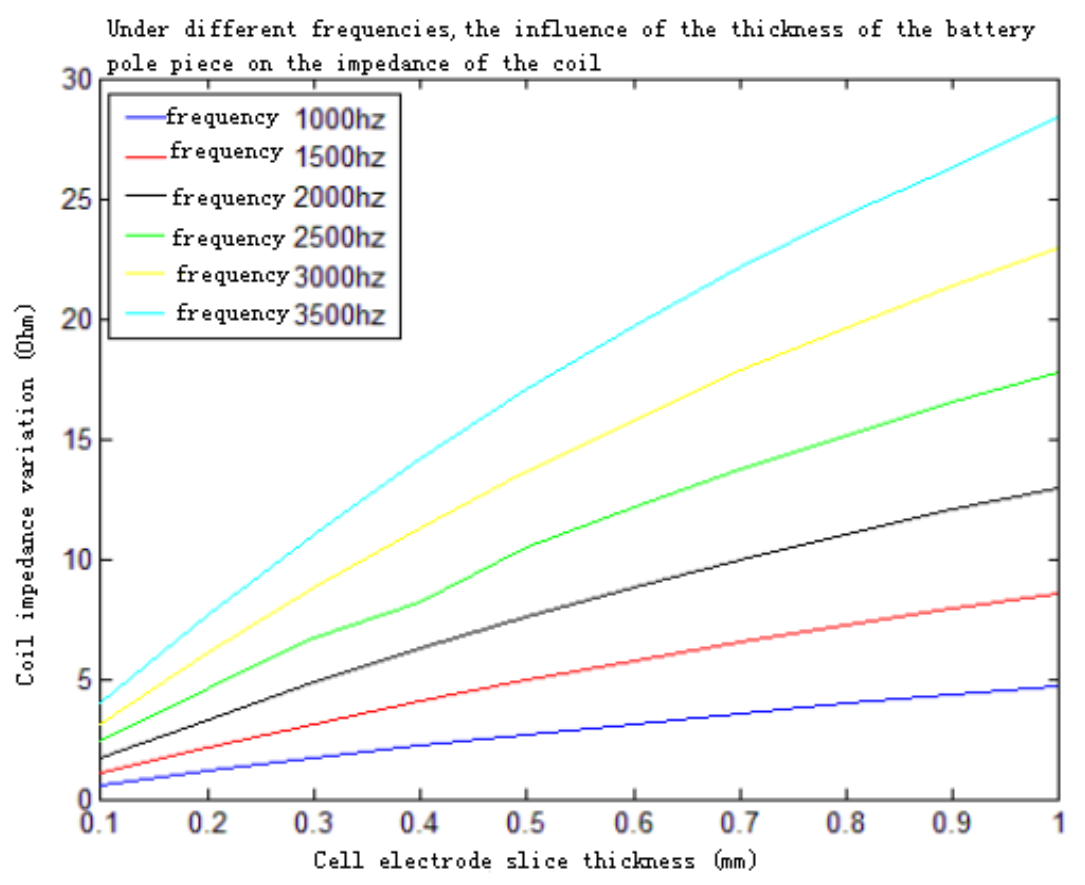

Fig. 3.6

Can be seen from figure 3.6,the variation of the coil impedance increases with the increase of the thickness of the cell.But at different excitation frequency, the change of the coil impedance is different.When the excitation frequency is high, the variation of the coil impedance increases with the increase of the thickness of the sheet metal, and the variation degree is larger, and the data deviation may be larger; when the excitation frequency is low, the variation of the coil impedance increases with the increase of the thickness of the sheet metal, the change is small, which is beneficial to the accuracy of the data.So in the experiment, we can improve the accuracy of data measurement by reducing the excitation frequency.

\section{Full summary}

This paper focuses on the measurement of the thickness of the battery pole piece,the theory and method of eddy current nondestructive testing is introduced,Discussion and Research on the measurement of the thickness of the battery pole based on eddy current.

The main research contents of this paper are summarized as follows:

(1)The history, research status and development trend of eddy current testing are studied;

(2)The basic principle of eddy current testing is studied,the main factors that affect the coil impedance of eddy current testing are studied by means of impedance analysis,and the calculation formula of the coil impedance is derived;

(3)Based on Matlab software, the program to measure the impedance of the coil is studied and designed, which is convenient for the follow-up study;

(4)By simulating the impedance of the coil, the eddy current testing of the battery pole piece can be obtained.In the case of other conditions unchanged, the change of the thickness of the battery pole piece can be obtained by studying the impedance variation of the coil impedance,so as to detect the density uniformity of the battery pole piece.

\section{References}

[1]XiaojingXu,BaihuaDuan,XuanhuiQu,PingLi.Research progress of thermal battery electrode materials,J.Rare Metals \& Cemented Carbides.(2006) 
[2]XiaoyuYuan,WeiminMa,LeiWen,HuarenYang,Research progress of thermal battery cathode materials,J.Journal of Ceramics.(2010)

[3]XiangXie, AijunLiu,Basic principle and application method of thermal battery,J.Power Supply Technologies and Applications.(1999)

[4]HaifengSun;JunZhuo,Research progress of thermal battery electrode materials,J.Materials Review.(2013)

[5]JinChong, JunjiCao,JinfengZhao, ShubenDong, WeihongZhang,JingDong,JinchengZhu,

Thermal battery cathode material,J.Chinese Journal of Power Sources.(2004)

[6]LinlinZhao,PingZhao,HongGao,XiaohuiCao,DadongHan,New progress of research on thermal battery cathode materials,J.Chinese Battery Industry.(2010)

[7]YuminLi,Factors affecting the electrical performance of thermal battery,J.Journal of Detection \& Control.(2001)

[8]GuirongLiu,ZhiyunPu,LingWang,YanbinPei,Study on density uniformity of fine grain tungsten alloy,J.Powder Metallurgy Industry.(2010)

[9]WenwenShan,BaoheYuan,JiayunXu, LinZhang,YanhongWang,Control of foam density uniformity of Polyacrylate,J.High Power Laser and Particle Beams.(2010)

[10]ChuandongWang,Review of the development of thermal battery,J.Chinese Journal of Power Sources.(2013)

[11]ZhiyouLi, XuanhuiQu,BaiyunHuang,Research progress of thermal battery,J.Battery Bimonthly.(1997)

[12]HongHou, ChunXiaoZhang,Design and evaluation of thermal battery reliability,J.Aviation Weapon.(2010) 